Slavica

bruxellensia

\section{Slavica bruxellensia}

Revue polyphonique de littérature, culture et histoire

slaves

$2 \mid 2009$

Femme(s)

\title{
Extraits de Pobeg kumaniki / La Pousse de mûrier sauvage de Lena Eltang
}

Traduction du russe

\section{Lena Eltang}

Traducteur : Anne Delizée

\section{(QpenEdition Journals}

Édition électronique

URL : http://journals.openedition.org/slavica/168

DOI : 10.4000/slavica. 168

ISSN : 2034-6395

Éditeur

Université libre de Bruxelles - ULB

Édition imprimée

Pagination : 57-64

ISSN : 2031-7654

Référence électronique

Lena Eltang, « Extraits de Pobeg kumaniki / La Pousse de mûrier sauvage de Lena Eltang », Slavica bruxellensia [En ligne], 2 | 2009, mis en ligne le 19 juin 2012, consulté le 19 avril 2019. URL : http:// journals.openedition.org/slavica/168; DOI : 10.4000/slavica.168

\section{(c) ())}

Les contenus de Slavica bruxellensia sont mis à disposition selon les termes de la Licence Creative Commons Attribution - Pas d'Utilisation Commerciale - Pas de Modification 3.0 France. 


\section{Traduction :}

\section{Lena Eltang \\ Побег куманики \\ La pousse de ronce \\ (Extraits)}

La mise en page, la ponctuation et la typographie respectent celles de l'original.

\section{PREMIÈRE PARTIE. L'ANGE SUR LE SABLE. CHORA}

(pp. 9-10)

MORAS

septembre, 17

à quoi je pense?

ma propriétaire, señora pardes, regarde dans mon armoire et touche mon linge

j'avais caché une coccinelle dans le tiroir des caleçons, et au soir elle n'y était plus

il faudrait parler à la patrona, lorsque je me souviendrai de l'espagnol

avant je savais toutes les langues du monde, même le ndembu, et après j'ai oublié

le docteur dit que mes ennuis sont dus à l'amour des mots

l'autre docteur m'a dit que je devais écrire un journal intime, chaque jour que dieu fait, d'écrire tout ce que je pense

ça demande beaucoup trop de mots, ils perlent sur les lèvres en gros sel, résonnent dans la tête en bourdons dorés, s'émiettent en lait glacé, en crabes transparents s'éparpillent sur le sable, en faibles micas de libellule volètent au vent, encrassent l'écoulement d'eau en épaisse manne céleste, comme les plaies de la douce dryade s'assèchent en liquide ambré, mais si je cesse d'écrire, tout disparaîtra

c'est vrai, non, docteur?

septembre, 17, le soir

odi et amo

encore à l'hôpital j'ai remarqué que les médecins te considèrent avec tendresse lorsqu'ils savent que tu guériras, c'est un peu comme si se détendait en eux un ressort impitoyable, tu n'es plus simplement un estado desesperado, un 
copeau de réalité, juste bon à allumer le feu, tu n'es pas encore leur égal, mais tu es déjà quelque chose d'autre

de fortuit et de faible tu te dresses en tension et constance et voilà déjà que les walkyries tissent l'étoffe de la victoire, faisant passer dans la chaîne de ta chair une habile navette de flèches rouges, et les médecins te regardent comme des dieux repus, et dansent joyeusement sous leurs couronnes de roseaux, moi ils me regardaient autrement - ça, je m'en souviens bien, bien que j'aie carrément oublié beaucoup de choses

(...)

DEUXIÈME PARTIE. L'ANGE DE VERRE. SUPPRESSION DE L'ACCIDENTEL

(pp.184-186)

To: Mr. Chanchal Prahlad Roy,

Sigmund-Haffner-Gasse 6 A-5020 Salzburg

From: Dr. Jonatan Silzer York,

Golden Tulip Rossini, Dragonara Road,

St Julians STJ 06, Malta

Februar, 26

Chanchal, mon coeur ! Les événements prennent une teinte de plus en plus sinistre, je commence à me dire qu'il faudrait mieux partir d'ici et rester plus longtemps chez ma mère à Hallein. S'il n'y avait pas cette maudite interdiction de quitter le territoire national que j'ai signée chez l'enquêteur, c'est sans doute ce que je ferais dans les prochains jours.

Il y a trois jours, le Français est mort - tu te souviens, celui qui avait la drôle de manie de la persécution ? -, et de la manière la plus absurde qui soit, je dois dire.

On a trouvé son corps dans le port, sur un chantier, il est tombé dans une tranchée où se trouvaient de grosses bobines de câble métallique ou quelque chose du genre. On dit qu'il courait et qu'il a trébuché, mais où donc, je te le demande, peut-on courir sur un territoire portuaire encombré de déchets de chantier, et ce, dans l'obscurité la plus totale ? (...)

Même le fait que ce pauvre Eugène avait volé un pot en terre cuite appartenant à l'expédition - ou personnellement à Oscar Theo Forge, je ne m'y retrouve plus - et avait selon toute vraisemblance essayé de le vendre, ne diminue pas pour moi le degré tragique de cette histoire.

J'ignore les détails, mais je pense qu'on me convoquera chez l'enquêteur après le professeur et Fiona, qui passent ces derniers jours plus de temps à la police qu'autre part. (...)

Fiona est une petite dame pour le moins fort intéressante. À mon retour je te raconterai quelques histoires piquantes... ce n'est pas que ses aventures me passionnent, c'est simplement amusant d'observer comment une rousse maigrichonne qui ne paie pas de mine mène en bateau trois apollons hétérosexuels, dont aucun n'essuierait de refus de ma part. 
J'espère que par ce passage je t'ai obligé à être ne fût-ce qu'un peu jaloux ?

Le quinze février Levah et moi avons été interrogés ensemble à propos de la mort de l'Anglaise, il agitait les bras et était terriblement indigné, il disait qu'on lui interdisait de quitter Malte.

Ah oui, tu ne sais rien de l'Anglaise, cher Chanchal.

Je ne voulais pas te tracasser, mais maintenant ça n'a plus d'importance, einerlei. (...)

Réponds-moi donc au plus vite, Chanchal, mon enfant.

Je t'envoie un paquet d'arachides sucrées, qu'elles au moins effleurent ta bouche magnifique.

\section{NOTES D'OSCAR THEO FORGE}

Malte, six mars

En combien de parties était divisée cette maudite matière première ? Joann n'écrit rien à ce propos, rien de rien. Il faut reconnaître que je l'ai ajouté à sa place. De ma propre voix.

Lesdits objets sont rassemblés en fonction du nombre d'esprits élémentaires, du nombre de victimes et de maîtres, du nombre de clés et du nombre de planètes. Les maîtres détenteurs des objets prodigieux s'élèveront chacun vers sa forme parfaite à celle fin du fruit précieux, lequel recevra le dernier.

Ça, c'est on ne peut plus clair. N'importe quel processus suppose certaines étapes. Il existe le début de l'opus et sa fin. Au cours de l'œuvre a lieu la distribution des cadeaux.

Je voudrais savoir à quelle étape nous nous trouvons actuellement.

Ou plutôt non, je ne le voudrais pas. Tout ce que je veux à présent, c'est m'allonger sur le tapis dans mon appartement londonien, poser la tête sur les genoux de Nadia et fermer les yeux. La pauvre chérie, elle a sans doute entrepris des travaux par ennui, comme la dernière fois, lorsque j'étais resté planté trois mois dans le Minnesota, je ne serais pas étonné de trouver à mon retour des étagères chinoises dans toute la maison et des rideaux de soie à lotus. 


\section{JOURNAL INTIME DE PETRA GROFF}

Voilà encore des pièces pour le puzzle que tout le monde a déjà laissé tomber, même Accroid me rit au nez lorsque je commence à en parler.

- Ma chère Petra, m'a-t-il dit hier lorsque nous buvions un café au Tropical, laisse O.T.Forge tranquille, il est trop coriace pour toi. Si la rousse irlandaise et lui sont vraiment trempés dans une affaire louche, ce n'est pas notre problème. Tu as envoyé un rapport à la commission de sauvegarde des monuments, ils n'ont qu'à les prendre au collet eux-mêmes, s'ils en ont le temps. Ce n'est pas une affaire criminelle, tu comprends ?

- Cependant, trois cadavres en deux mois..., ai-je commencé, mais il ne m'a même pas laissé parler, il a déposé un billet de dix sur la table et il est parti. Il semble que j'aie de nouveau commencé à grossir.

Mais voici à quoi je pense.

Après la mort de Nadia, son père a guéri sur-le-champ, ça fait un. Dommage qu'elle ne l'ait pas su. Après la mort du Français, on a appris qu'il était plein aux as. C'est sûr que ça ne lui a pas servi à grand-chose.

Après le suicide du docteur, la clinique à Salzbourg a été fermée. Je suppose qu'il le souhaitait de tout son cœur après qu'ils l'avaient jeté à la rue. C'est Fiona qui m'a raconté à propos du docteur, mais je n'y avais pas fait attention. Maintenant je sais que j'aurais dû. Il s'ensuit que tout ce que ces gens voulaient s'est réalisé dès qu'ils sont morts, d'une manière ou d'une autre.

L'histoire est digne de mon ex-amie Véronique.

Du candomblé brésilien avec maracas du début à la fin. Ça lui aurait plu.

Mais que veut donc le professeur O.T.Forge?

Et l'étudiant-macédonien veut-il quelque chose ? Et moi, qu'est-ce que je veux d'eux tous?

Pourquoi ne pas écouter Accroid?

(...)

(p.278)

\section{MORAS}

sans date

trop de duchesses

alors, en mars, fiona est arrivée toute mouillée, et nous nous sommes réchauffés près de la cuisinière au gaz et parlions d'écureuils, c'est-à-dire qu'elle parlait - moi je n'avais rien à dire, tout ce que je sais sur les écureuils tient en une ligne de l'ancienne edda : la discorde entre l'aigle et le dragon sur l'arbre yggdrasil 
tu vois, toute notre équipe tiendrait sur ce frêne, riait fiona, moi je suis le médiateur roux qui sème la discorde sans le vouloir, oscar l'aigle pétri d'amourpropre, jonatan le faucon déplumé perché entre les yeux de l'aigle, le français et gustave les cerfs continuellement affamés aux ramures de chêne, quant à la pauvre nadia, la chèvre heidrun, bien sûr !

et qui est alors le dragon qui est dans les racines, j'ai demandé, le dragon, c'est toi ! allons donc, quel dragon, je serais encore d'accord pour balder, le gars qui est mort d'une pousse de gui, ou bien pour un pot de miel sacré, mais fiona est intraitable

(...)

TROISIÈME PARTIE. L'ANGE DE PIERRE. APOKATASTASIS

(pp.317-320)

\section{From: Dr Fiona Russell russellfiona@hotmail.com To: Gustop Zemeroj (imprimer pour le professeur Forge)}

Je vais tenter d'exposer mes réflexions de manière ordonnée.

La matière a produit cinq éléments sombres, dit le papyrus.

Ces éléments s'opposent aux cinq éléments clairs créés par l'Homme primordial. Ainsi, à l'air s'oppose la fumée, au feu source de chaleur la flamme destructrice, à l'eau pure s'oppose le brouillard, au vent frais le cruel tourbillon.

À la lumière éclatante s'opposent, bien sûr, les ténèbres.

Je vois ici une coïncidence évidente avec notre conten $u^{1}$, tant dans sa réalisation matérielle que mystique.

Je vous en prie, ne rejetez pas ma lettre sous le coup de la colère, lisez jusqu'au bout, il ne s'agit ici ni d'orgueil scientifique ni de droit de cuissage, il s'agit de la mort de trois personnes dans un moulin à viande pour lequel nous avions le mode d'emploi d'une machine à coudre. Pardon si je suis grossière et péremptoire, ce n'était pas facile pour moi de me décider à vous écrire cette lettre, et maintenant je m'en fais terriblement.

Commençons par le premier jour, lorsqu'est morte la pauvre Nadia. Nous avons conclu que la cause de sa mort était l'artéfact air, c'est-à-dire la flèche à pointe de fer et à tête d'aigle gravée sur la hampe. L'aigle personnifie l'élément air, il est le roi de l'espace, l'oiseau tantrique Garuda, avez-vous déclaré.

Vous vous souvenez, j'ai polémiqué, j'ai parlé des Sumériens pour qui l'aigle est le symbole du soleil, l'oiseau d'Assur, de I'aigle syrien aux bras humains, personnifiant l'adoration du soleil, de la symbolique chinoise et grecque associant I'aigle à l'oiseau solaire, mais vous avez campé sur vos positions, cher professeur. L'air, voilà tout. 
Et je sais pourquoi! Vous aviez sur les bras six objets, dont chacun devait correspondre à un des six éléments.

Et vous n'avez simplement pas vu pour la flèche d'autre explication, sauf peut-être le symbole alchimique du soufre et la définition hindouiste de la flèche en tant que le dard de la mort.

Mais si nous oublions l'inévitable jeu des six éléments distribué par Joann le retors croupier, le tableau change immédiatement et impitoyablement, cher Oscar.

Le roi des mondes du Vent est à l'image de l'aigle.

Son corps est de fer, et le corps de tous ceux qui appartiennent

Au Vent est de fer.

Leur goût est piquant sous n'importe quelle forme.

C'est ce qu'écrivent les Kephalaia à propos de l'élément du vent implacable, qui s'oppose à la légère brise vitale ; c'est la description du démon du monde du Tourbillon, et elle représente admirablement l'arme à la pointe de fer qui a tué votre amie. C'est son corps qui l'a tuée: le fer. Le mouvement du carreau d'arbalète, engendrant le vent mortel. Excusez-moi, je vous en prie, de vous obliger à vous souvenir de cet insupportable matin de février.(...)

Votre F.

(...)

(pp.354-355)

\section{MORAS}

sans date

reasons

aujourd'hui on m'a dit qu'au soir viendrait le barbier et qu'il couperait les cheveux de tout le monde, mais je ne veux pas, je ne le ferai pas

j'ai quatre raisons à cela

si je suis sumérien, mes cheveux ressemblent à la fumée, ils protègent des esprits mauvais

si je suis shiva, mes cheveux ébouriffés sont le symbole de mon renoncement

si à moi, comme à isis pleurant osiris, on me coupe une touffe blanche, la douleur perdra sa force

je connais en vérité le secret de la boucle qui orne le front de l'enfant dieu, dit un mort du livre des morts, moi aussi je sais, mais si je leur dis, ils se mettront de nouveau à échanger des regards

mais Absalon le révolté, dit franca la rusée catholique, s'est emmêlé les cheveux dans les branches d'un chêne et on l'a tué

oui, mais Samson en revanche, je lui dis, en plus j'appartiens à une autre confession 
et les prêtres égyptiens, dit franca, ils sont bien rasés

ce sont les prêtres, je dis, les enfants de sang royal, eux, portaient une tresse sur le côté droit de la tête, et pour t'horrifier jusqu'au bout, je te dirai que le nom horus signifie l'enfant à la touffe épaisse

laisse-moi, dalila 